\title{
Suppressed Ion Migration along the In-Plane Direction in Layered Perovskites
}

\author{
Xun Xiao, ${ }^{\dagger, \perp \odot}$ Jun Dai, ${ }^{\ddagger}, \perp \odot$ Yanjun Fang, ${ }^{\S}$ Jingjing Zhao, ${ }^{\dagger, \S}$ Xiaopeng Zheng, ${ }^{\S}$ Shi Tang, ${ }^{\S}$ \\ Peter Neil Rudd, ${ }^{\dagger}$ Xiao Cheng Zeng, ${ }^{\ddagger 0}$ and Jinsong Huang ${ }^{*}, \dagger, \S_{0}$ \\ ${ }^{\dagger}$ Department of Applied Physical Sciences, University of North Carolina, Chapel Hill, North Carolina 27599, United States \\ ${ }^{\ddagger}$ Department of Chemistry, University of Nebraska-Lincoln, Lincoln, Nebraska 68588, United States \\ ${ }^{\S}$ Department of Mechanical and Materials Engineering, University of Nebraska-Lincoln, Lincoln, Nebraska 68588, United States
}

ABSTRACT: Ion migration in a three-dimensional (3D) perovskite is the source of many unique phenomena such as photocurrent hysteresis and a giant switchable photovoltaic effect and can also accelerate the degradation of perovskite-based electronic devices. Here we report the observation of suppressed ion migration along the in-plane direction of layered perovskites by studying the conductivity of layered single-crystal perovskites at varied temperatures. Large-area layered perovskite thin single crystals are synthesized by the space-confined method. The absence of ion migration in these layered perovskites can be explained by an increase in the energy required to form an ion vacancy, compared to 3D perovskites. The suppressed ion migration in layered perovskites indicates that they have intrinsically better stability under an electric field and may contribute to the improved perovskite stability in devices made of layered perovskite through the reduction of ion diffusion-induced perovskite degradation or corrosion of charge transport layers and electrodes.

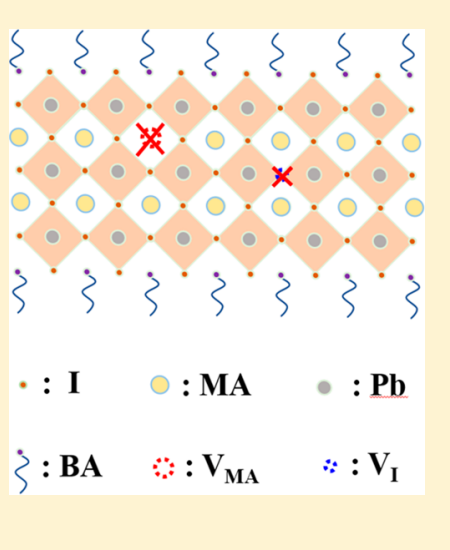

$\mathrm{O}$ rganic-inorganic hybrid perovskite (OIHP) materials have aroused tremendous interest as one of the most promising candidates for the next generation of solar cells due to their auspicious optoelectronic properties, such as large light absorption coefficient, long carrier diffusion lengths, ${ }^{1}$ and high carrier mobility. ${ }^{2-4}$ These properties have led to the power conversion efficiency (PCE) of OIHP solar cells quickly reaching the parity of silicon solar cells after only a few years of development. ${ }^{5,6}$ However, one major hindrance to the commercialization of OIHP solar cells is the relatively low stability compared to their silicon counterparts. In particular, OIHP is found to be sensitive to moisture, oxygen, heat, as well as ultraviolet light. ${ }^{7-9}$ Apart from these environmental factors, ion diffusion-induced corrosion of the charge transport layer or metal electrode is also capable of causing device failure. ${ }^{10,11}$

Many studies have been done to enhance the stability of OIHP solar cells by addressing the various external stimuli. ${ }^{4,12,13}$ The application of encapsulation method has been used to protect the OIHP active layer from moisture ${ }^{9}$ and oxygen. $^{14,15}$ In order to address the corrosion of transport layers by ion diffusion, corrosion-stopping layers have also been developed to maintain separation between the OIHP layers and the metal electrodes. ${ }^{16,17}$ Now that the external stimuli can be largely negated by sophisticated device encapsulation techniques, the intrinsic stability of OIHP needs more attention to even further enhance the stability of perovskite solar cells. ${ }^{18}$ Recent studies show very promising device stability under illumination for a few months while even reaching 1 year with some perovskite materials by adding quasi-two-dimensional (quasi-2D) perovskite. ${ }^{19-21}$ This raises one fundamental question as to why quasi-2D perovskites are intrinsically more stable than conventional three-dimensional (3D) perovskites like $\mathrm{MAPbI}_{3} \cdot{ }^{19,22,23}$ Several mechanisms have been proposed to explain the enhanced moisture and thermal stability of quasi-2D perovskites or materials with quasi-2D perovskites. It is intuitive that the hydrophobic organic side chain in quasi-2D perovskites could prevent direct exposure to moisture. ${ }^{13}$ The improved structural stability has been attributed to stronger van der Waals interactions between the long organic side chain and the $\left[\mathrm{PbI}_{6}\right]$ units. Improving these interactions means that more energy is needed to decompose the quasi-2D structure by removing the organic molecules, compared to methylammonium cations $\left(\mathrm{MA}^{+}\right)$or formamidinium cations $\left(\mathrm{FA}^{+}\right)$in $3 \mathrm{D}$ perovskite. ${ }^{24,25}$ In addition, ion migration has been shown to be one main origin of the intrinsic instability of 3D halide perovskites. ${ }^{18}$ Our recent study shows that the long-chain organic ligands could form a barrier preventing ion migration along the out-of-plane direction. ${ }^{26,27}$ However, ions in OIHPs may also migrate along the in-plane direction, i.e., along the layer in quasi-2D perovskite, where

Received: January 11, 2018

Accepted: February 14, 2018

Published: February 14, 2018 
$\mathbf{a}$

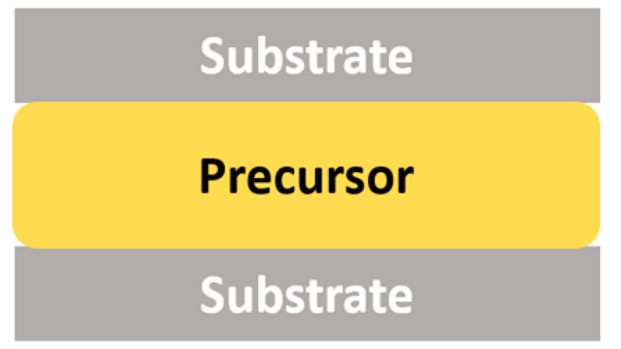

C
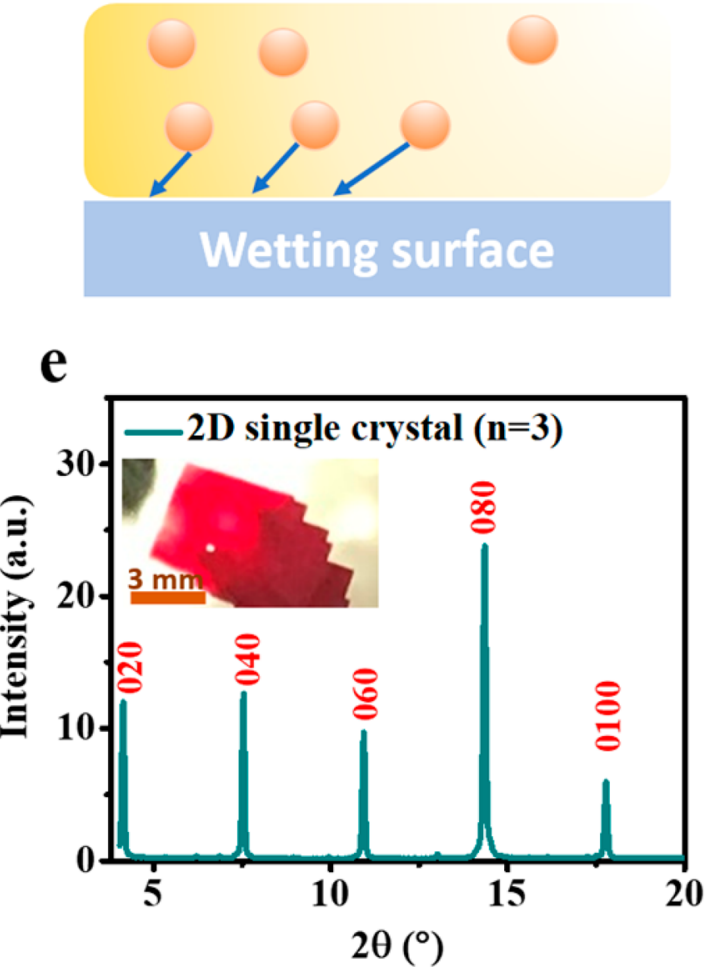

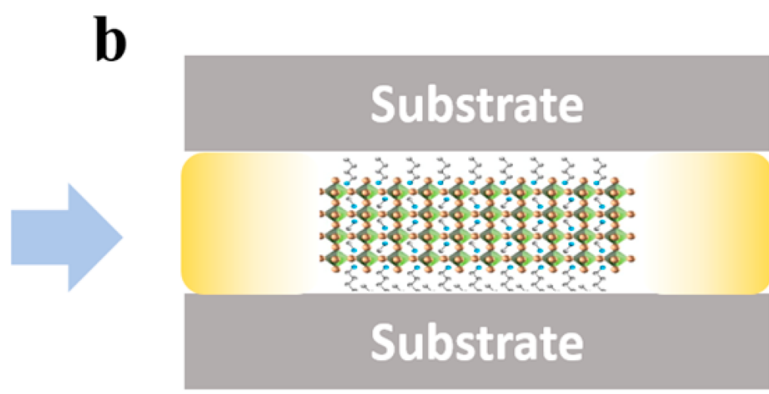

d

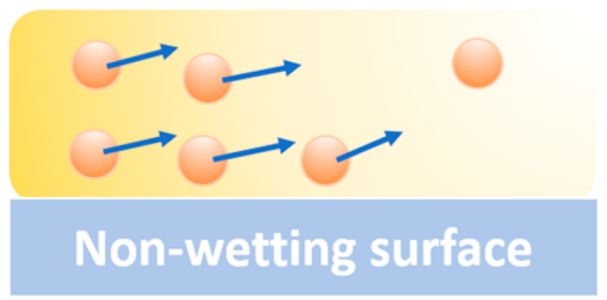

f

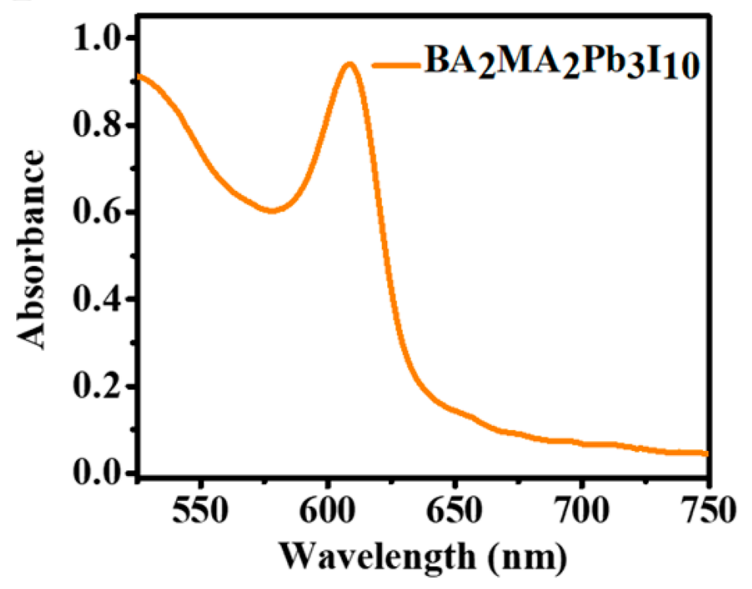

Figure 1. (a,b) Schematic illustration of the space-confined method for the growth of $\mathbf{B A}_{2} \mathbf{M A}_{2} \mathrm{~Pb}_{3} \mathrm{I}_{10}$ single crystals; schematic illustration of ion transportation in the confined space on $(c)$ wetting and $(d)$ nonwetting surfaces, (e) XRD of a quasi-2D single crystal $(n=3)$; the inset is a photograph of the quasi-2D single crystal; ( $f)$ absorption of an exfoliated quasi-2D $B_{2} M_{2} A_{2} P_{3} I_{10}(n=3)$ single crystal.

organic ligands would not be capable of blocking transportation pathways. $^{22}$ Because the current conduction in quasi-2D perovskites is along the in-plane direction, due to the large resistance of the long organic ligands, the ion migration along these layers needs to be understood to determine how their intrinsic stability is limited by ion migration. ${ }^{28}$

Here, we investigate the intrinsic ion migration stability in the layers of quasi-2D perovskites. By using temperaturedependent conductivity measurements, it was found that the ion migration along the in-plane direction is suppressed. The origin of suppressed ion migration in the layered perovskites is determined to be a lower density of point defects such as iodide vacancies $\left(\mathrm{V}_{\mathrm{I}}\right)$ and methylammonium vacancy $\left(\mathrm{V}_{\mathrm{MA}}\right)$. The calculated formation energies of $\mathrm{V}_{\mathrm{I}}$ and $\mathrm{V}_{\mathrm{MA}}$ in RuddlesdenPopper-type layered perovskite $\mathrm{BA}_{2} \mathrm{MA}_{2} \mathrm{~Pb}_{3} \mathrm{I}_{10}(n=3)$ are larger than that in $\mathrm{MAPbI}_{3}$, resulting in the suppression of point defect formation.

To characterize the intrinsic ion migration properties of layered perovskites, single crystals of Ruddlesden-Popper-type $\mathrm{BA}_{2} \mathrm{MA}_{2} \mathrm{~Pb}_{3} \mathrm{I}_{10}(n=3)$ were synthesized using a space-confined method to produce large-area perovskite single crystals with controlled thickness, as shown in Figure 1a,b. ${ }^{29}$ Typically, two nonwetting substrates were used to construct the confined space, and the saturated perovskite solution was inserted into the space, as shown in Figure 1a. The single crystals were grown by cooling-induced supersaturation. As illustrated in Figure $1 c, d$, the introduction of a nonwetting surface could enhance the long-range ion transport along the confined space due to the reduced dragging-force of the solvent molecules with which perovskite ions have formed a complex. ${ }^{30,31}$ As the solution was gradually cooled, $\mathrm{BA}_{2} \mathrm{MA}_{2} \mathrm{~Pb}_{3} \mathrm{I}_{10}(n=3)$ single crystals grew between the nonwetting substrates, as illustrated in Figure $1 \mathrm{~b}$. In the perovskite precursor solution, perovskite ions would form complexes with solvent, which meant that the diffusion rate of perovskite ions was determined by the diffusion speed of solvent. The nonwetting surface weakened the solvent-substrate interaction, accelerating the transportation of solvent. ${ }^{29}$ As a result, the long-range ion diffusion along the confined space could be achieved, allowing for the growth of large quasi-2D $\left(\mathrm{BA}_{2} \mathrm{MA}_{2} \mathrm{~Pb}_{3} \mathrm{I}_{10}\right)$ single crystals. This 
a

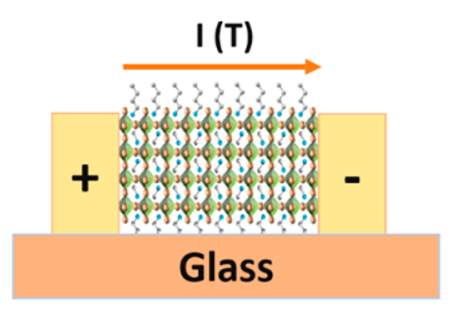

b

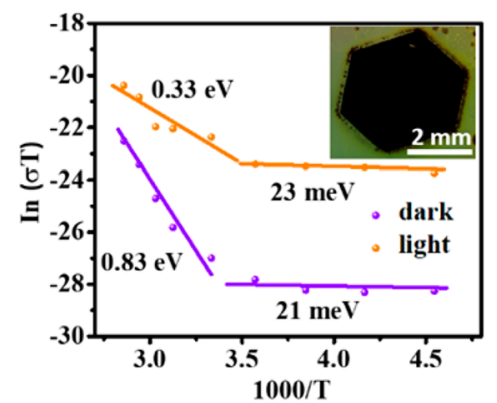

c

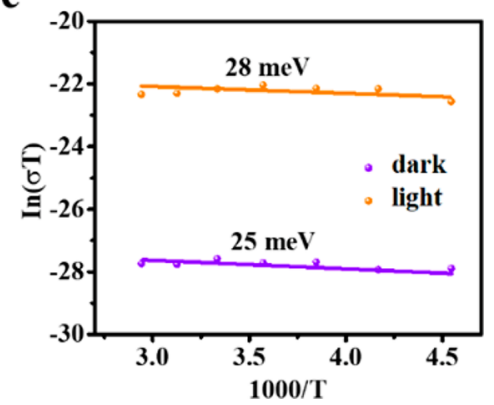

Figure 2. (a) Scheme for the in-plane temperature-dependent conductivity of a quasi-2D $\mathrm{BA}_{2} \mathrm{MA}_{2} \mathrm{~Pb}_{3} \mathrm{I}_{10}$ perovskite; (b,c) results of temperature-dependent conductivity measurement of $3 \mathrm{D}$ and quasi-2D perovskite single crystals, respectively. The inset in (b) is a photo of a $\mathrm{MAPbI}_{3}$ single crystal.

is also the first time that large-area quasi-2D perovskite thin single crystals are reported to be synthesized using a spaceconfined method. The photograph of the single crystal is shown in the inset of Figure 1e. The single crystals grown using this technique have a typical lateral size of $4 \mathrm{~mm}$ and a thickness of $20 \mu \mathrm{m}$. The observed absorption edge at approximately $650 \mathrm{~nm}$ and excitation peak at $\sim 610 \mathrm{~nm}$, seen in Figure 1f, are indicative that the grown single crystal is, in fact, a $\mathrm{BA}_{2} \mathrm{MA}_{2} \mathrm{~Pb}_{3} \mathrm{I}_{10}(n=3)$ quasi-2D perovskite. X-ray diffraction (XRD) was used to analyze the phase structure and orientation of the $\mathrm{BA}_{2} \mathrm{MA}_{2} \mathrm{~Pb}_{3} \mathrm{I}_{10}$ single crystal. As shown in Figure 1e, all diffraction peaks in the XRD pattern are indexed to be $(0 k 0)$ planes of $\mathrm{BA}_{2} \mathrm{MA}_{2} \mathrm{~Pb}_{3} \mathrm{I}_{10}$, confirming the orientation of the quasi-2D single crystal and indicating that the perovskite layers are parallel to the substrate. ${ }^{19}$

To understand the intrinsic ion migration stability of these layered perovskites, we studied the ion migration activation energy in perovskite layers, determined by measuring the inplane electric conductivity of the quasi-2D BA $\mathrm{BA}_{2} \mathrm{~Pb}_{3} \mathrm{I}_{10}(n=$ 3) single crystals at varied temperatures. To complete the measurements, electrodes were deposited at the edges of the quasi-2D single crystal to make lateral electrical contact for a lateral device structure, as shown in Figure 2a. The lateral device structure is introduced to suppress the electronic conduction and highlight the ionic conduction contribution to the total conductivity by the geometry effect. Within the lowtemperature region, the ion concentration is very small and the contribution of ion migration to conductivity is negligible, and thus, electronic conductivity dominates the material conductivity. However, in the high-temperature region, the number of ions that are capable of migration increases dramatically, meaning that the ionic conductivity would dominate the conductivity in the high-temperature region. By measuring the temperature-dependent electrical conductivity, we could derive the ion activation energy $\left(E_{\mathrm{A}}\right)$ from the electric conductivity in the high-temperature region using the Nernst-Einstein relation $^{32}$

$$
\sigma(T)=\frac{\sigma_{0}}{T} \exp \left(-\frac{E_{\mathrm{A}}}{k_{\mathrm{B}} T}\right)
$$

The activation energy could be derived from the slope of the $\ln (\sigma \mathrm{T})-1 / k T$ relation. In the measurement, a constant electrical field was set to be $0.2 \mathrm{~V} / \mu \mathrm{m}$, which was small to reduce the poling effect. Figure $2 \mathrm{~b}$ shows the total electrical conductivity of single-crystal $\mathrm{MAPbI}_{3}$ with a lateral size of 3 $\mathrm{mm}$ and thickness of $\sim 14 \mu \mathrm{m}$, as shown in the inset of Figure
$2 \mathrm{~b}$ in the dark and under illumination. For the 3D $\mathrm{MAPbI}_{3}$ single crystal, conductivity in the low-temperature region $(T<$ $280 \mathrm{~K}$ ) was ascribed to the electronic conduction, while it was dominated by ionic conductivity in the high-temperature region $(T>280 \mathrm{~K})$. The activation energy at high temperature $(T>$ $280 \mathrm{~K}$ ) was fitted to be $0.83 \mathrm{eV}$ in dark conditions, which is consistent with our previous results. ${ }^{32}$ The activation energy was reduced to $0.33 \mathrm{eV}$ under 0.25 sun illumination, quantitatively showing ion migration in $\mathrm{MAPbI}_{3}$ to be much easier under illumination. ${ }^{32}$ In the low-temperature region $(T<$ $280 \mathrm{~K}$ ), the activation energy was fitted to be $22 \pm 2 \mathrm{meV}$, which was within the shallow charge trap depth range for electronic conduction. For the 3D perovskite single crystals, a clear transition from electronic to ionic conduction is observed in Figure $2 b$, both in the dark and under illumination. However, for quasi-2D $\mathrm{BA}_{2} \mathrm{MA}_{2} \mathrm{~Pb}_{3} \mathrm{I}_{10}$ perovskite samples, we did not observe such a transition, regardless of whether the samples were in the dark or under illumination (Figure 2c). A constant slope with an activation energy of $25 \pm 3 \mathrm{meV}$ was observed, representative of the electronic conduction while also indicating that the carrier trap depth is similar to that of 3D perovskites. The absence of transition from electronic conduction to ionic transition in this measurement indicates that ion migrationinduced current along the in-plane direction is also negligible up to $350 \mathrm{~K}$ in the quasi-2D $\mathrm{BA}_{2} \mathrm{MA}_{2} \mathrm{~Pb}_{3} \mathrm{I}_{10}$ single crystals.

To elucidate the origin of suppressed ion migration along the layers in the quasi-2D $\mathrm{BA}_{2} \mathrm{MA}_{2} \mathrm{~Pb}_{3} \mathrm{I}_{10}$ single crystals, we examined the defect formation as the presence of defects should be necessary to mediate ion migration. Because grain boundaries are not available in quasi-2D single crystals, ions have to migrate with the help of vacancies in the quasi-2D layers. Density functional theory (DFT) calculation, as implemented in the VASP program, was conducted to calculate the formation energies of $\mathrm{V}_{\mathrm{I}}$ and $\mathrm{V}_{\mathrm{MA}}$. These vacancies prove to be most important to consider as they are the two main types of ions that may migrate in halide perovskites. ${ }^{33}$ The generalized gradient approximation in the form of PerdewBurke-Ernzerhof (PBE) was used for the exchangecorrelation functional. The ion-electron interaction was treated with the projector-augmented wave (PAW) method. Grimme's DFT-D3 correction was adopted to describe the long-range van der Waals interactions. The vacancy defects were modeled by removing an $\mathrm{I}^{-}\left(\right.$or $\left.\mathrm{MA}^{+}\right)$ion from a $2 \times 2 \times$ $2 \mathrm{MAPbI}_{3}$ supercell or $2 \times 2$ quasi- $2 \mathrm{D} \mathrm{BA}_{2} \mathrm{MA}_{2} \mathrm{~Pb}_{3} \mathrm{I}_{10}$ supercell. The defect formation energy was calculated as $E_{\mathrm{f}}=E(A)+$ $E(B)-E(A B)$, where $\mathrm{A}$ stands for $\mathrm{MAPbI}_{3}$ or quasi-2D $\mathrm{BA}_{2} \mathrm{MA}_{2} \mathrm{~Pb}_{3} \mathrm{I}_{10}$ with a vacancy, $\mathrm{B}$ represents a single vacancy, 
and $\mathrm{AB}$ is pristine $\mathrm{MAPbI}_{3}$ or quasi- $2 \mathrm{D} \mathrm{BA}_{2} \mathrm{MA}_{2} \mathrm{~Pb}_{3} \mathrm{I}_{10}$. The calculated formation energies of $\mathrm{V}_{\mathrm{MA}}$ are 5.72 and $6.94 \mathrm{eV}$ in $\mathrm{MAPbI}_{3}$ and quasi-2D $\mathrm{BA}_{2} \mathrm{MA}_{2} \mathrm{~Pb}_{3} \mathrm{I}_{10}$, respectively, while the calculated formation energies of $\mathrm{V}_{\mathrm{I}}$ are 3.44 and $5.46 \mathrm{eV}$ in $\mathrm{MAPbI}_{3}$ and quasi-2D $\mathrm{BA}_{2} \mathrm{MA}_{2} \mathrm{~Pb}_{3} \mathrm{I}_{10}$, respectively. The significantly increased formation energies of $V_{I}$ and $V_{M A}$ in quasi-2D perovskites result in a much lower vacancy density relative to that of $3 \mathrm{D}$ perovskite. The reduced bulk point defect density has been frequently reported in low-dimensional nanomaterials, which is also favored in thermodynamics. ${ }^{34}$ The quasi-2D perovskites have strong analogies to quantum dots, as evidenced by the excitonic peaks in absorption spectra. ${ }^{34}$ As illustrated in Figure 3a, vacancies are one major

a

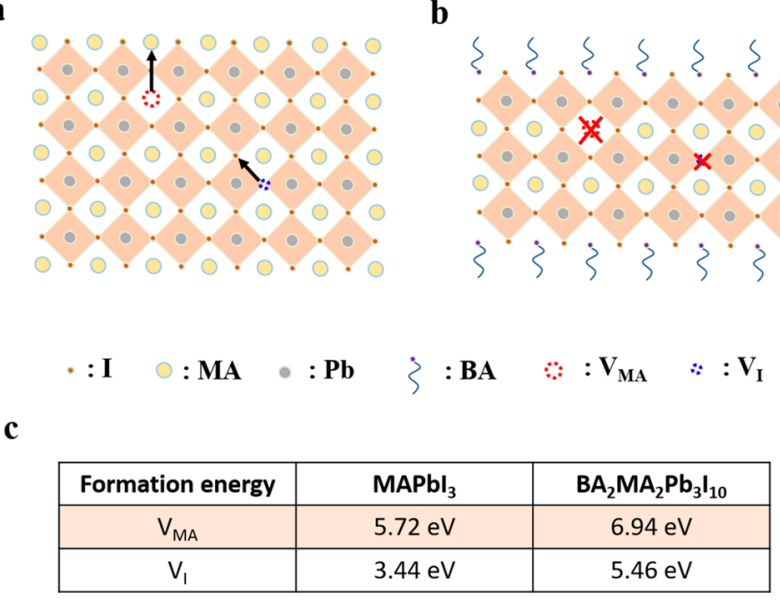

Figure 3. Schemes of the vacancy path for ion migration in 3D (a) and quasi-2D (b) perovskites and (c) formation energy of vacancy defects $\left(V_{M A}, V_{I}\right)$ in $3 D$ and quasi-2D perovskites.

trigger of ion migration in 3D perovskite as vacancy sites allow for a pathway along which ions could effectively migrate. ${ }^{35}$ According to the substitution diffusion model, ion diffusion can only occur if there is a vacancy at an adjacent lattice point for the ion to jump to. The diffusion of ions occurring depends on (1) whether the atoms have sufficient thermal energy to overcome the activation energy barrier to migrate $\left(\Delta G_{\mathrm{m}}\right)$ and (2) the probability that there is an adjacent vacancy, which is given by $z X_{\mathrm{V}}$ (where $\mathrm{z}$ is the number of nearest neighbors, and $X_{V}$ is the probably that one site is vacant). The increase of $V_{M A}$ and $V_{I}$ formation energies, resulting in a lower density of vacancies, would subsequently form a larger energy barrier for ions to migrate and thus enhance the intrinsic ion migration stability as well as structural stability.

The absence of ion migration and reduction of vacancy defects along the sheets of quasi-2D perovskite should impact the efficiency and stability of perovskite-based electronic devices, including solar cells, photodetectors, as well as light emitting diodes (LEDs). ${ }^{13,25,26}$ For instance, photocurrent hysteresis as well as light-induced phase separation in OIHP solar cells could be suppressed in quasi-2D perovskite due to the suppression of ion migration and higher structural stability. ${ }^{4,36}$ For photodetectors, decreasing the number of defects would largely solve $1 / f$ noise, which comes from the trapping and detrapping process, and thus, a low noise detector could be achieved. ${ }^{37}$ In addition, because LED devices are working with bias on, the suppression of ion migration would efficiently stabilize the structure under external electric field, thus improving the working lifetime of OIHP LED devices. ${ }^{23}$
In conclusion, vacancy defects including $V_{M A}$ and $V_{I}$ were found to be far more difficult to form in quasi-2D perovskites, which reduced the vacancy sites for ions to migrate. Thus, ion migration along the electric channel in quasi-2D perovskites was suppressed. The absence of ion migration in quasi-2D perovskites should provide a promising opportunity for improved device efficiencies and device stability. The implementation of qausi-2D perovskites would effectively suppress the ion migration-induced decomposition of perovskite, degradation of charge transport layers, and corrosion of metal electrodes.

\section{ASSOCIATED CONTENT}

\section{Supporting Information}

The Supporting Information is available free of charge on the ACS Publications website at DOI: 10.1021/acsenergylett.8b00047.

Growth of single crystals, device fabrication process, and DFT calculations (PDF)

\section{AUTHOR INFORMATION}

\section{Corresponding Author}

*E-mail: jhuang@unc.edu.

ORCID $\odot$

Xun Xiao: 0000-0002-9810-2448

Jun Dai: 0000-0001-6599-8826

Xiao Cheng Zeng: 0000-0003-4672-8585

Jinsong Huang: 0000-0002-0509-8778

\section{Author Contributions}

${ }^{\perp}$ X.X. and J.D. contributed equally.

\section{Notes}

The authors declare no competing financial interest.

\section{ACKNOWLEDGMENTS}

This work was supported by the Office of Naval Research (N00014-17-1-2727) and National Science Foundation (OIA1538893 and DMR-1420645).

\section{REFERENCES}

(1) Shi, D.; Adinolfi, V.; Comin, R.; Yuan, M.; Alarousu, E.; Buin, A.; Chen, Y.; Hoogland, S.; Rothenberger, A.; Katsiev, K.; et al. Low trapstate density and long carrier diffusion in organolead trihalide perovskite single crystals. Science 2015, 347 (6221), 519-522.

(2) Dong, Q.; Fang, Y.; Shao, Y.; Mulligan, P.; Qiu, J.; Cao, L.; Huang, J. Electron-hole diffusion lengths $>175 \mu \mathrm{m}$ in solution-grown CH3NH3PbI3 single crystals. Science 2015, 347 (6225), 967-970.

(3) Wang, Q.; Shao, Y.; Dong, Q.; Xiao, Z.; Yuan, Y.; Huang, J. Large fill-factor bilayer iodine perovskite solar cells fabricated by a lowtemperature solution-process. Energy Environ. Sci. 2014, 7 (7), 23592365.

(4) Shao, Y.; Xiao, Z.; Bi, C.; Yuan, Y.; Huang, J. Origin and elimination of photocurrent hysteresis by fullerene passivation in $\mathrm{CH} 3 \mathrm{NH} 3 \mathrm{PbI} 3$ planar heterojunction solar cells. Nat. Commun. 2014, $5,5784$.

(5) Kojima, A.; Teshima, K.; Shirai, Y.; Miyasaka, T. Organometal halide perovskites as visible-light sensitizers for photovoltaic cells. J. Am. Chem. Soc. 2009, 131 (17), 6050-6051.

(6) Zheng, X.; Chen, B.; Dai, J.; Fang, Y.; Bai, Y.; Lin, Y.; Wei, H.; Zeng, X. C.; Huang, J. Defect passivation in hybrid perovskite solar cells using quaternary ammonium halide anions and cations. Nat. Energy 2017, 2, 17102.

(7) Yang, J.; Siempelkamp, B. D.; Liu, D.; Kelly, T. L. Investigation of $\mathrm{CH} 3 \mathrm{NH} 3 \mathrm{PbI} 3$ degradation rates and mechanisms in controlled 
humidity environments using in situ techniques. ACS Nano 2015, 9 (2), 1955-1963.

(8) Yang, S.; Wang, Y.; Liu, P.; Cheng, Y.-B.; Zhao, H. J.; Yang, H. G. Functionalization of perovskite thin films with moisture-tolerant molecules. Nat. Energy 2016, 1, 15016.

(9) Christians, J. A.; Miranda Herrera, P. A.; Kamat, P. V. Transformation of the excited state and photovoltaic efficiency of $\mathrm{CH}_{3} \mathrm{NH}_{3} \mathrm{PbI}_{3}$ perovskite upon controlled exposure to humidified air. $J$. Am. Chem. Soc. 2015, 137 (4), 1530-1538.

(10) Mateker, W. R.; McGehee, M. D. Progress in understanding degradation mechanisms and improving stability in organic photovoltaics. Adv. Mater. 2017, 29, 1603940.

(11) Han, Y.; Meyer, S.; Dkhissi, Y.; Weber, K.; Pringle, J. M.; Bach, U.; Spiccia, L.; Cheng, Y.-B. Degradation observations of encapsulated planar $\mathrm{CH} 3 \mathrm{NH} 3 \mathrm{PbI} 3$ perovskite solar cells at high temperatures and humidity. J. Mater. Chem. A 2015, 3 (15), 8139-8147.

(12) Lee, J. W.; Kim, D. H.; Kim, H. S.; Seo, S. W.; Cho, S. M.; Park, N. G. Formamidinium and cesium hybridization for photo-and moisture-stable perovskite solar cell. Adv. Energy Mater. 2015, 5 (20), 1501310.

(13) Smith, I. C.; Hoke, E. T.; Solis-Ibarra, D.; McGehee, M. D.; Karunadasa, H. I. A layered hybrid perovskite solar-cell absorber with enhanced moisture stability. Angew. Chem. 2014, 126 (42), 1141411417.

(14) Li, X.; Tschumi, M.; Han, H.; Babkair, S. S.; Alzubaydi, R. A.; Ansari, A. A.; Habib, S. S.; Nazeeruddin, M. K.; Zakeeruddin, S. M.; Grätzel, M. Outdoor performance and stability under elevated temperatures and long-term light soaking of triple-layer mesoporous perovskite photovoltaics. Energy Technology 2015, 3 (6), 551-555.

(15) Aristidou, N.; Eames, C.; Sanchez-Molina, I.; Bu, X.; Kosco, J.; Islam, M. S.; Haque, S. A. Fast oxygen diffusion and iodide defects mediate oxygen-induced degradation of perovskite solar cells. Nat. Commun. 2017, 8, 15218.

(16) Zhao, J.; Zheng, X.; Deng, Y.; Li, T.; Shao, Y.; Gruverman, A.; Shield, J.; Huang, J. Is $\mathrm{Cu}$ a stable electrode material in hybrid perovskite solar cells for a 30-year lifetime? Energy Environ. Sci. 2016, 9 (12), 3650-3656.

(17) Dong, X.; Fang, X.; Lv, M.; Lin, B.; Zhang, S.; Ding, J.; Yuan, N. Improvement of the humidity stability of organic-inorganic perovskite solar cells using ultrathin $\mathrm{Al}_{2} \mathrm{O}_{3}$ layers prepared by atomic layer deposition. J. Mater. Chem. A 2015, 3 (10), 5360-5367.

(18) Zhao, J.; Deng, Y.; Wei, H.; Zheng, X.; Yu, Z.; Shao, Y.; Shield, J. E.; Huang, J. Strained hybrid perovskite thin films and their impact on the intrinsic stability of perovskite solar cells. Sci. Adv. 2017, 3 (11), eaao5616.

(19) Cao, D. H.; Stoumpos, C. C.; Farha, O. K.; Hupp, J. T.; Kanatzidis, M. G. 2D homologous perovskites as light-absorbing materials for solar cell applications. J. Am. Chem. Soc. 2015, 137 (24), $7843-7850$.

(20) Grancini, G.; Roldán-Carmona, C.; Zimmermann, I.; Mosconi, E.; Lee, X.; Martineau, D.; Narbey, S.; Oswald, F.; De Angelis, F.; Graetzel, M.; et al. One-Year stable perovskite solar cells by 2D/3D interface engineering. Nat. Commun. 2017, 8, 15684.

(21) Wang, N.; Cheng, L.; Ge, R.; Zhang, S.; Miao, Y.; Zou, W.; Yi, C.; Sun, Y.; Cao, Y.; Yang, R.; et al. Perovskite light-emitting diodes based on solution-processed self-organized multiple quantum wells. Nat. Photonics 2016, 10 (11), 699-704.

(22) Wang, Z.; Lin, Q.; Chmiel, F. P.; Sakai, N.; Herz, L. M.; Snaith, H. J. Efficient ambient-air-stable solar cells with 2D-3D heterostructured butylammonium-caesium-formamidinium lead halide perovskites. Nat. Energy 2017, 2 (9), 17135.

(23) Yuan, M.; Quan, L. N.; Comin, R.; Walters, G.; Sabatini, R.; Voznyy, O.; Hoogland, S.; Zhao, Y.; Beauregard, E. M.; Kanjanaboos, P.; Lu, Z.; Kim, D. H.; Sargent, E. H. Perovskite energy funnels for efficient light-emitting diodes. Nat. Nanotechnol. 2016, 11, 872-877.

(24) Quan, L. N.; Yuan, M.; Comin, R.; Voznyy, O.; Beauregard, E. M.; Hoogland, S.; Buin, A.; Kirmani, A. R.; Zhao, K.; Amassian, A.; et al. Ligand-stabilized reduced-dimensionality perovskites. J. Am. Chem. Soc. 2016, 138 (8), 2649-2655.
(25) Zhang, X.; Ren, X.; Liu, B.; Munir, R.; Zhu, X.; Yang, D.; Li, J.; Liu, Y.; Smilgies, D.-M.; Li, R.; et al. Stable high efficiency twodimensional perovskite solar cells via cesium doping. Energy Environ. Sci. 2017, 10 (10), 2095-2102.

(26) Tsai, H.; Nie, W.; Blancon, J.-C.; Stoumpos, C. C.; Asadpour, R.; Harutyunyan, B.; Neukirch, A. J.; Verduzco, R.; Crochet, J. J.; Tretiak, S.; et al. High-efficiency two-dimensional RuddlesdenPopper perovskite solar cells. Nature 2016, 536 (7616), 312-316.

(27) Lin, Y.; Bai, Y.; Fang, Y.; Wang, Q.; Deng, Y.; Huang, J. Suppressed Ion Migration in Low Dimensional Perovskites. ACS Energy Letters 2017, 2 (7), 1571-1572.

(28) Xiao, Z.; Yuan, Y.; Shao, Y.; Wang, Q.; Dong, Q.; Bi, C.; Sharma, P.; Gruverman, A.; Huang, J. Giant switchable photovoltaic effect in organometal trihalide perovskite devices. Nat. Mater. 2015, 14 (2), 193-198.

(29) Chen, Z.; Dong, Q.; Liu, Y.; Bao, C.; Fang, Y.; Lin, Y.; Tang, S.; Wang, Q.; Xiao, X.; Bai, Y.; et al. Thin single crystal perovskite solar cells to harvest below-bandgap light absorption. Nat. Commun. 2017, 8 (1), 1890 .

(30) Sendner, C.; Horinek, D.; Bocquet, L.; Netz, R. R. Interfacial water at hydrophobic and hydrophilic surfaces: Slip, viscosity, and diffusion. Langmuir 2009, 25 (18), 10768-10781.

(31) Wang, Q.; Shao, Y.; Xie, H.; Lyu, L.; Liu, X.; Gao, Y.; Huang, J. Qualifying composition dependent $\mathrm{p}$ and $\mathrm{n}$ self-doping in $\mathrm{CH}_{3} \mathrm{NH}_{3} \mathrm{PbI}_{3}$. Appl. Phys. Lett. 2014, 105 (16), 163508.

(32) Xing, J.; Wang, Q.; Dong, Q.; Yuan, Y.; Fang, Y.; Huang, J. Ultrafast ion migration in hybrid perovskite polycrystalline thin films under light and suppression in single crystals. Phys. Chem. Chem. Phys. 2016, 18 (44), 30484-30490.

(33) Yuan, Y.; Huang, J. Ion migration in organometal trihalide perovskite and its impact on photovoltaic efficiency and stability. Acc. Chem. Res. 2016, 49 (2), 286-293.

(34) Tang, J.; Kemp, K. W.; Hoogland, S.; Jeong, K. S.; Liu, H.; Levina, L.; Furukawa, M.; Wang, X.; Debnath, R.; Cha, D.; et al. Colloidal-quantum-dot photovoltaics using atomic-ligand passivation. Nat. Mater. 2011, 10 (10), 765-771.

(35) Yuan, Y.; Chae, J.; Shao, Y.; Wang, Q.; Xiao, Z.; Centrone, A.; Huang, J. Photovoltaic switching mechanism in lateral structure hybrid perovskite solar cells. Adv. Energy Mater. 2015, 5 (15), 1500615.

(36) Hoke, E. T.; Slotcavage, D. J.; Dohner, E. R.; Bowring, A. R.; Karunadasa, H. I.; McGehee, M. D. Reversible photo-induced trap formation in mixed-halide hybrid perovskites for photovoltaics. Chem. Sci. 2015, 6 (1), 613-617.

(37) Peng, W.; Yin, J.; Ho, K.-T.; Ouellette, O.; De Bastiani, M.; Murali, B.; El Tall, O.; Shen, C.; Miao, X.; Pan, J.; et al. Ultralow SelfDoping in Two-dimensional Hybrid Perovskite Single Crystals. Nano Lett. 2017, 17 (8), 4759-4767. 